\title{
Coherent synchrotron radiation free longitudinal bunch shaping using transverse deflecting cavities
}

\author{
Gwanghui Ha๑, ${ }^{1,}{ }^{*}$ John G. Power, ${ }^{1}$ Jiahang Shao, ${ }^{1}$ Manoel Conde, ${ }^{1}$ and Chunguang Jing ${ }^{2}$ \\ ${ }^{1}$ Argonne National Laboratory, Lemont, Illinois 60439, USA \\ ${ }^{2}$ Euclid Techlabs LLC, Bolingbrook, Illinois 60440, USA
}

(Received 26 March 2020; accepted 24 June 2020; published 8 July 2020)

\begin{abstract}
Longitudinal bunch shaping is gaining increasing attention due to the large impact it has on modern accelerators. The existing methods for controlling the density profile fall into three categories: laser shaping, emittance exchange, or correlations between the energy and one of the other coordinates. These methods require either (i) dispersive elements (e.g., dipole magnet), which generate coherent synchrotron radiation, or (ii) shaping near the cathode, which can distort the profile due to space-charge effects. These collective effects significantly decrease shaping quality as the bunch charge increases. None of the existing methods are currently capable of shaping high-charge bunches. In this paper, we introduce a transverse deflecting cavity-based shaping method to avoid the impact of collective effects on the shaping process. In this method, a transverse deflecting cavity introduces the $z-x$ correlation, and a transverse mask tailors the longitudinal density profile. This method provides high-quality shaping regardless of charge level and preserves the beam quality.
\end{abstract}

DOI: 10.1103/PhysRevAccelBeams.23.072803

\section{INTRODUCTION}

Over the past decade, the electron linear accelerator community has explored several methods of longitudinal bunch shaping (hereafter, shaping) with the common goal of accurate shaping over a broad ranges of bunch parameters (charge, bunch length, etc.) while maintaining beam quality. However, in all of the shaping methods introduced so far, both the shaping and the beam quality decrease as the bunch charge increases. The major limit on the quality comes from the collective effects that appear in these shaping methods. These previous shaping methods can be categorized into three groups: laser shaping, emittance exchange (EEX), and correlation-based methods, which we describe below. Each method is intrinsically connected to collective effects, either coherent synchrotron radiation (CSR) or space charge (SC).

The first method, laser-based shaping [1-3], works by shaping the longitudinal profile of the incident laser pulse that impinges on the photocathode to generate an electron bunch. Because the laser determines the longitudinal position $(z)$ of these electrons, it is possible to shape an electron bunch's longitudinal profile using this method.

\footnotetext{
"gha@anl.gov
}

Published by the American Physical Society under the terms of the Creative Commons Attribution 4.0 International license. Further distribution of this work must maintain attribution to the author(s) and the published article's title, journal citation, and DOI.
Although this method has the advantage of the shaping technology being located outside of the accelerator vacuum, its shaping quality is limited to low charge due to the strong SC forces near the cathode [2,3], which tends to smear out the desired electron profile.

The second method, EEX-based shaping, first shapes the transverse dimension of the bunch and is followed by an exchange of the transverse and longitudinal phase spaces to create the desired longitudinal profile $[4,5]$. Although this method can generate high-quality shapes at moderate charges, the EEX beam line requires dipole magnets. Therefore, the electron bunch passing through the dipole magnet generates CSR, which significantly deteriorates the shaping quality and the emittance of the bunch at high charge. Although there has been progress on improving the shaping quality [6] and suppressing emittance growth [7], CSR is still the major limiting factor of this method.

The third method, correlation-based shaping, introduces a correlation between an easy-to-control axis and the target axis. It applies a manipulation to the control axis, which simultaneously affects the target axis. There are two different correlation-based methods used to shape the bunch's longitudinal profile. The first manipulates the bunch's energy distribution along the $z$ axis using rf cavities followed by an nonisochronous beam line (e.g., chicane), which provides a nonzero $R_{56}$. This $R_{56}$ correlates the $z$ and energy $(\delta)$ distribution of the bunch and shapes the longitudinal density distribution as a result [8-10]. The second correlation-based method uses a dispersive beam 
line to introduce a correlation between the transverse position $(x)$ and $(\delta)$. If a bunch has an initial $z-\delta$ correlation due to $\mathrm{rf}$ curvature or SC force, a transverse mask to change $\delta$ distribution affects $z$ distribution at the same time [11]. These correlation-based methods (either $R_{56}$ or dispersion) require dispersive elements (e.g., a dipole magnet), and, therefore, the bunch experiences CSR at high charge.

To overcome the limitations of the collective effects present in existing shaping methods, we introduce a transverse deflecting cavity (TDC)-based shaping method. This shaping concept was inspired by our earlier work on gigahertz laser-free time-resolved transmission electron microscopy [12] and a similar concept proposed in Ref. [13]. The TDC-based shaping method can be broken down into three main steps, which are described in more detail in the remainder of the paper. First, TDC generates a correlation between the transverse momentum $\left(x^{\prime}\right)$ and $z$ through the $B_{y}$ component of its dipole field, which then evolves into an $x-z$ correlation in the drift space following the TDC. Second, a transverse mask is applied at this location to shape the longitudinal density profile. Third, the remaining correlation is eliminated with additional optics. Because TDC-based shaping takes place in a straight line, this method is free from CSR. In addition, if the bunch's energy is high enough for its charge level, SC can be ignored, too. Thus, TDC-based shaping at a high enough energy is free from collective effects.

In Sec. II, we describe the principle of the TDC-based shaping method, its shaping quality, and the various TDCshaping beam line configurations that can be used to implement the method. In Sec. III, we provide start-toend simulation results to illustrate the performance of the method, as well as its limitation. Because this method will be a significant step forward for beam-driven wakefield accelerators, we highlight three applications for beamdriven wakefield accelerators in Sec. IV: improved transformer ratio for high-charge bunches, improved efficiency using main beam shaping, and high-charge, high-frequency bunch trains for terahertz two-beam acceleration $(\mathrm{THz}$ TBA). TDC shaping will, of course, impact many other branches of accelerator physics other than beamdriven wakefield acceleration, such as radiation generation. We briefly describe its application to radiation generation in Sec. II, but we leave details of those topics for future work.

\section{TDC-BASED SHAPING}

\section{A. Principle}

To explain the principle of TDC-based shaping, we use an example beam line that consists of three elements (two TDCs and a quadrupole magnet) and a transverse mask, all separated by drifts as shown in Fig. 1. The function of the first part of the beam line (from the entrance to immediately after the mask) is to project the longitudinal distribution of the beam into the horizontal space and then use the mask to shape the longitudinal distribution. The function of the second part of the beam line (from immediately after the mask to the exit) is to remove the unwanted correlations introduced by the first part of the beam line.

Given that the particle's coordinate at the $n$th element of the beam line is $\mathbf{X}_{n} \equiv\left(x_{n}, x_{n}^{\prime}, y_{n}, y_{n}^{\prime}, z_{n}, \delta_{n}\right)$, its transport through the first TDC can be described as [14]

$$
\left[\begin{array}{c}
x_{1, \text { ext }} \\
x_{1, \text { ext }}^{\prime} \\
y_{1, \text { ext }} \\
y_{1, \text { ext }}^{\prime} \\
z_{1, \text { ext }} \\
\delta_{1, \text { ext }}
\end{array}\right]=\left[\begin{array}{cccccc}
1 & L_{c} & 0 & 0 & \frac{L_{c}}{2} \kappa & 0 \\
0 & 1 & 0 & 0 & \kappa & 0 \\
0 & 0 & 1 & L_{c} & 0 & 0 \\
0 & 0 & 0 & 1 & 0 & 0 \\
0 & 0 & 0 & 0 & 1 & 0 \\
\kappa & \frac{L_{c}}{2} \kappa & 0 & 0 & \frac{12 N^{2} L_{c}}{1+2 N^{2}} \kappa^{2} & 1
\end{array}\right]\left[\begin{array}{c}
x_{1, \text { ent }} \\
x_{1, \text { ent }}^{\prime} \\
y_{1, \text { ent }} \\
y_{1, \text { ent }}^{\prime} \\
z_{1, \text { ent }} \\
\delta_{1, \text { ent }}
\end{array}\right] \text {, }
$$

where $L_{c}$ is the physical length of TDC, $\kappa\left(=\frac{e V_{\perp}}{p c} \frac{2 \pi}{\lambda}\right)$ is a normalized kick strength, $N$ is the number of cells in the cavity, and the subscripts ent and ext correspond to the entrance and exit of the element, respectively. We label the distance of the drift from the TDC to the mask as $L_{m}$. The particle's horizontal position at the mask $\left(x_{m}\right)$ becomes a function of the initial longitudinal position $\left(z_{1, \text { ent }}\right)$ :

$$
x_{m}=x_{1, \text { ent }}+\left(L_{m}+L_{c}\right) x_{1, \text { ent }}^{\prime}+\kappa\left(L_{m}+\frac{L_{c}}{2}\right) z_{1, \text { ent }} .
$$

Using quadrupole magnets located upstream of the first TDC (not shown in Fig. 1), one can focus the beam to the mask location such that the first two terms in Eq. (2) are minimized. In this case, $x_{m}$ is predominantly determined by $z_{1, \text { ent }}$, thus accomplishing the projection of the longitudinal distribution into the $x$ position. The mask can now be used
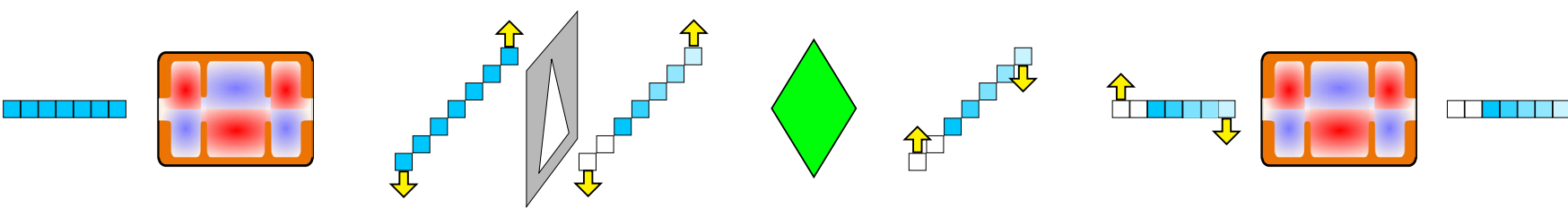

FIG. 1. Conceptual drawing to show the principle of TDC-based shaping. Rectangular boxes represent an electron bunch along the $z$ direction. Colors correspond to electron density. Yellow arrows show the direction of horizontal momentum $\left(x^{\prime}\right)$. 
to tailor the particle density along the longitudinal direction due to the $x_{m}-z_{1, \text { ent }}$ correlation we introduced. We use a mask similar to those in Refs. $[4,6]$ (see simplified diagram in Fig. 1), where the $y$ width of the mask is used to continuously control the longitudinal density. Appendix B contains more information from the start-to-end simulation.

The longitudinal shaping itself has been accomplished with the first part of the beam line, and we use the second part of the beam line (from immediately after the mask to the exit) to eliminate the remaining correlations, $x-z$ and $x^{\prime}-z$, because they increase the emittance in both directions and the tilted beam is not appropriate in most accelerator applications. We introduced two additional elements downstream of the mask to eliminate these remaining correlations, as shown in Fig. 1. The quadrupole flips the direction of $x^{\prime}$ to eliminate the horizontal offset generated by the kick from the TDC plus drift before the mask. Once the $x-z$ correlation has been eliminated in this way, a second TDC eliminates the $x^{\prime}-z$ correlation just as the first TDC generated the correlation.

We next derive conditions to eliminate the remaining correlations using matrix formalism, as shown in Eq. (1). If we name the distance between the $i$ th and $j$ th element $L_{i j}$, the particle's horizontal position and momentum before the second TDC are related to its initial coordinate as

$$
\begin{gathered}
x_{3, \text { ent }}=x_{1, \text { ent }}+L x_{1, \text { ent }}^{\prime} \\
+\kappa\left[L-\frac{L_{23}}{f_{1}}\left(L_{12}+\frac{L_{c}}{2}\right)\right] z_{1, \text { ent }}, \\
x_{3, \text { ent }}^{\prime}=x_{1, \text { ent }}^{\prime}+\kappa\left[1-\frac{1}{f_{1}}\left(L_{12}+\frac{L_{c}}{2}\right)\right] z_{1, \text { ent }},
\end{gathered}
$$

where $L$ is $L_{12}+L_{23}+\frac{L_{c}}{2}$ and $f_{1}$ is the focal length of the quadrupole. We do not count mask as an element here, since it does not change the optics. Thus, $n=2$ and $n=3$ correspond to the quadrupole and the second TDC, respectively, in Fig. 1. Note that we approximate the quadrupole as a thin lens. If the focal length satisfies the condition

$$
f_{1}=\frac{\left(L_{12}+\frac{L_{c}}{2}\right)\left(L_{23}+\frac{L_{c}}{2}\right)}{L_{12}+L_{23}+L_{c}}
$$

then the $x-z$ correlation can be removed. The remaining $x^{\prime}-z$ correlation shown in Eq. (4) can be eliminated when the kick from the second TDC is set to

$$
\kappa_{2}=\frac{2 L_{12}+L_{c}}{2 L_{23}+L_{c}} \kappa_{1} .
$$

Note that the correlation between the transverse and longitudinal directions is eliminated only at high energy so that the longitudinal dispersion in the drift is negligible,
$L_{12}+L_{23}+L_{c} \ll \gamma^{2}$. If it is not negligible due to low energy, correlations will remain, similar to the situation in Ref. [12]. It is possible to eliminate the longitudinal dispersion at low energy by modifying the layout to include four TDCs. In the remainder of this paper, we assume that the beam energy is high enough to ignore the longitudinal dispersion in the drift.

\section{B. Shaping quality}

In this section, we derive a resolution term $(\Delta z)$ to quantify the shaping quality. If the bunch incident on the TDC-shaping beam line has zero horizontal emittance, then the kick from the first TDC will generate a line in the $x-z$ phase space and the resolution would be $0 \mathrm{~m}$. This means the final longitudinal profile exactly follows the horizontal profile cut by the mask, without any aberration. However, realistic bunches have nonzero emittance and, therefore, generate a finite thickness on the $x-z$ particle distribution. As shown in Fig. 2, the mask cutting the bunch transversely generates a spread of particles on the $z$ coordinate with a width of $\Delta z$. This spread at each horizontal position results in aberrations on the shaped profile and determines the resolution of the shaping. Although the simulation is necessary to accurately estimate $\Delta z$ for the real beam, we can derive a convenient equation for $\Delta z$ using several assumptions.

Using the thin-TDC approximation and ignoring the vertical optics, the $\Delta z$ for a Gaussian beam with truncation to $n \sigma$ at the mask location is

$$
\begin{gathered}
\Delta z\left(x_{m}\right)=\frac{2 \varepsilon_{x, 0} \sigma_{z, 0}\left(L_{m}+L_{01}\right)}{\sigma_{x, 0} \sigma_{x, m}{ }^{2}} \sqrt{n^{2}{\sigma_{x, m}}^{2}-x_{m}{ }^{2}}, \\
\sigma_{x, m}{ }^{2}=\frac{\left(L_{m}+L_{01}\right)^{2} \varepsilon_{x, 0}{ }^{2}}{\sigma_{x, 0}{ }^{2}}+\kappa^{2} L_{m}^{2} \sigma_{z, 0}{ }^{2},
\end{gathered}
$$

where $L_{01}$ is the drift length from the last focusing element to the first thin TDC, $L_{m}$ is the drift length from the thin TDC to the mask, and 0 and $m$ correspond to locations right after the focusing element and the mask location, respectively (see Appendix A for the derivation). Because the
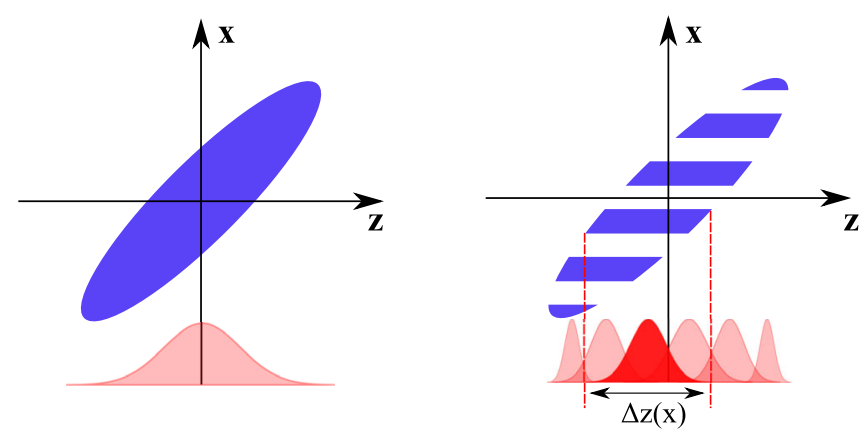

FIG. 2. Conceptual drawing to show shaping quality degradation due to nonzero emittance. 


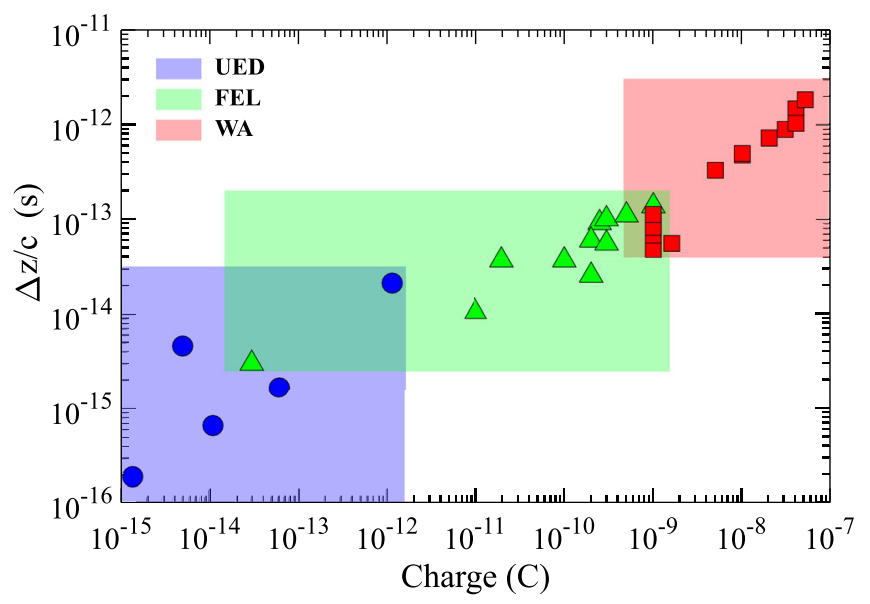

FIG. 3. $\Delta z$ calculated using beam parameters for existing facilities or design parameters from Refs. [15-28].

longitudinal terms should dominantly determine the size of the bunch at the mask location, $\sigma_{x, m}$ in Eqs. (7) and (8) can be approximated to $\kappa L \sigma_{z, 0}$. Then $\Delta z\left(x_{m}\right)$ can be simplified to

$$
\begin{aligned}
\Delta z\left(x_{m}\right)= & \frac{2 \varepsilon_{x, 0}}{\kappa \sigma_{x, 0}}\left(1+\frac{L_{01}}{L_{m}}\right) \sqrt{n^{2}-\left(\frac{x_{m}}{\sigma_{x, m}}\right)^{2}} \\
& =\frac{2 c\left(m_{e} c^{2}\right) \varepsilon_{n, x, 0}}{\omega_{R F} V_{\perp} \sigma_{x, 0}}\left(1+\frac{L_{01}}{L_{m}}\right) \sqrt{n^{2}-\left(\frac{x_{m}}{\sigma_{x, m}}\right)^{2}},
\end{aligned}
$$

where $\varepsilon_{n, x, 0}$ is the initial normalized emittance and $V_{\perp}$ is the kick voltage of TDC. Because this $\Delta z$ corresponds to the full spread, it will exaggerate the effect on the actual profile. If the distribution of the spread is close to Gaussian, it would be more accurate to use $\Delta z(0) / 6$ with $n=3$.

Equation (9) indicates TDC shaping's potential for highprecision shaping. We calculated $\Delta z$ using beam parameters from simulations and measurements for existing facilities to demonstrate its capabilities. Figure 3 shows calculated $\Delta z$. Each data point in the shaded block corresponds to a facility for ultrafast electron diffraction (UED), free-electron laser (FEL), or a wakefield accelerator (WA). For this calculation, we used $\sigma_{x, 0}$ of $1 \mathrm{~mm}$ for the UED area and $5 \mathrm{~mm}$ for FEL and WA. The TDC kick voltage was set to $5 \mathrm{MV}$ with $1.3 \mathrm{GHz}$ for all cases. $L_{m}$ and $L_{01}$ are chosen to be 1 and $0.3 \mathrm{~m}$, respectively. Although beam line settings are not optimized for each facility's beam parameters, $\Delta z$ already shows impressive values. According to Fig. 3, TDC-based shaping can potentially provide picosecond- or subpicosecond-level shaping quality for nanocoulomb to tens of nanocoulomb bunches and femtosecond-level quality for FEL or UED beams.

We will discuss WA applications in more detail in Sec. IV. However, note that high-quality shaping has other important applications relevant to both UEDs and FELs.
We can imagine applying this technique to generate a bunch train for superradiant radiation [29] or to generate two electron or two x-ray pulses that are precisely separated in time for pump-probe experiments [30]. Because the time separation will be determined by mechanically cut slits, there will be negligible jitter on the arrival timing between these two pulses. These two pulses separated in time can be applied for precision experiments.

Note that the use of a fixed mask means the longitudinal profile is vulnerable to the jitter of the transverse beam size at the mask location. For example, if the input beam has a uniform $x-y$ distribution at the mask location, the jitter will not affect on the output longitudinal profile unless the beam becomes smaller than the mask. On the other hand, if the beam has a hot spot near the mask boundary, then a significant change in the output profile will result when the beam size jitters. This should be considered during the shaping system design.

\section{Chirp control capability and other configurations}

In addition to bunch shaping, TDC-based shaping beam lines can be used to control the chirp of the bunch's longitudinal phase space, as proposed in Ref. [31]. The TDC not only kicks the bunch transversely but also accelerates or decelerates particles proportional to its transverse position due to its electric field of the dipole mode. When $x-z$ correlation exists in TDC, this electric field introduces energy change proportional to $z$ due to the $x-z$ correlation. To exploit this additional benefit, it is important to control $x-z$ correlation at TDCs.

In the case of the $2 \mathrm{TDC}+1 \mathrm{Q}$ configuration, as shown in the top row in Fig. 4, there is no $x-z$ correlation on the incident bunch. Because TDC introduces a weak $x-z$ correlation evolved from $x^{\prime}-z$ correlation that TDC generates as a bunch drifts in TDC with a finite length, this configuration can provide chirp control but in a narrow control range due to this weak $x-z$ correlation. In addition, this control depends on $\kappa_{1}$. However, it is not
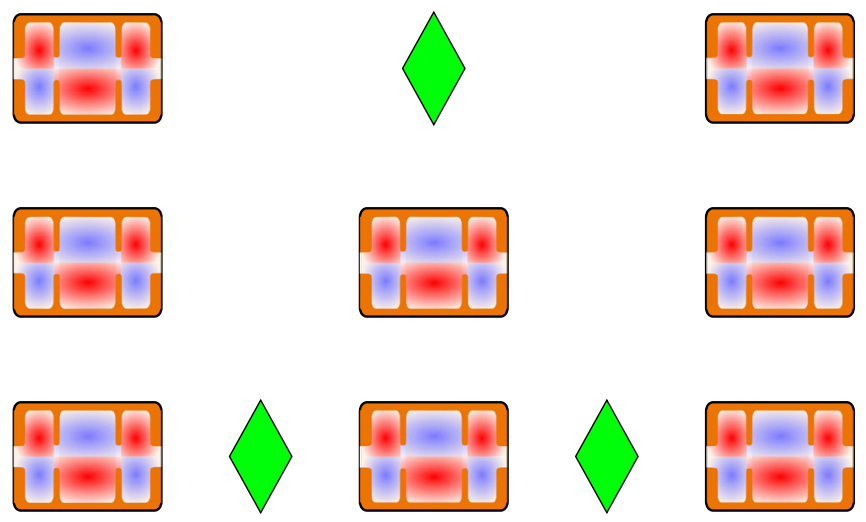

FIG. 4. Three different configurations provide time-transverse coupling-based shaping. 
preferable to change $\kappa_{1}$, because it is related to the shaping quality. This is why other configurations can provide extra benefits beyond the 2TDC + 1Q configuration.

\section{1. $3 T D C$ and $3 T D C+2 Q$ configurations}

In the previous discussion, the beam line configuration used was $2 \mathrm{TDC}+1 \mathrm{Q}$. However, this is not the only viable configuration. While all configurations start with a TDC and a mask, the downstream of beam line for controlling the remaining correlation can vary. Here we provide two additional configurations as examples.

The first beam line we consider is the TDC + TDC + TDC (3TDC) configuration (see the middle row in Fig. 4). The only difference is the replacement of the quadrupole in the middle of $2 \mathrm{TDC}+1 \mathrm{Q}$ configuration with another TDC. Instead of flipping the direction of $x^{\prime}$ using the quadrupole, one can use the second TDC, whose phase is $180^{\circ}$ off from the first cavity's. Because the timing between the particles does not change (at a high enough energy), the second TDC can flip the direction of $x^{\prime}$. The rest of the beam line shares the same principle as $2 \mathrm{TDC}+1 \mathrm{Q}$ to eliminate $x^{\prime}-z$ correlation. This configuration works when the $\kappa_{1}, \kappa_{2}$, and $\kappa_{3}$ for each TDC satisfy the relationship below:

$$
\kappa_{2}=-\frac{L_{12}+L_{23}+2 L_{c}}{L_{23}+L_{c}}
$$

$$
\kappa_{3}=\frac{L_{12}+L_{c}}{L_{23}+L_{c}} \kappa_{1} .
$$

In 3TDC configuration, the bunch has a strong $x-z$ correlation at the second TDC due to the time-dependent kick from the first TDC. Thus, the second TDC can provide significant changes on the energy. In addition, the chirp control depends on both $\kappa_{1}$ and $\kappa_{2}$, because they determine the strength of $x-z$ correlation and acceleration, respectively. However, one limitation in this configuration is these control parameters. $\kappa_{1}$ is again not preferable as the control parameter, because it determines the shaping quality. Although $\kappa_{2}$ can be used to control the chirp, it should satisfy Eq. (10), which is the relation between $\kappa_{2}$ and spacing. Installed beam line has a fixed spacing, so this configuration provides a wide range of final chirp for the accelerator designer, but the chirp is not tunable during operation.

The second example uses more elements to increase the degrees of freedom. Here we add two additional quadrupoles to the 3TDC configuration (see the bottom row in Fig. 4). In this way, while the first TDC still provides a strong $x-z$ correlation, the following quadrupole can control this $x-z$ correlation. This becomes an additional knob with which to control the chirp. Because the $x^{\prime}-z$ correlation can be eliminated by the rest of beam line, this knob from the quadrupole controls the chirp during operation. The beam line setting should satisfy the condition below to eliminate the $x^{\prime}-z$ correlation:

$$
\begin{gathered}
\kappa_{2}=-\frac{-\left(2 L_{12}+L_{c}\right)\left(L_{24}+L_{c}\right) \kappa_{1}+\left(2 L_{14}+3 L_{c}\right) f_{1} \kappa_{1}-\left(2 L_{45}+L_{c}\right) f_{1} \kappa_{3}}{f_{1}\left(2 L_{34}+L_{c}\right)}, \\
f_{2}=\frac{\left(2 L_{34}+L_{c}\right)\left(2 L_{45}+L_{c}\right) f_{1} \kappa_{3}}{\left(2 L_{12}+L_{c}\right)\left(2 L_{23}+L_{c}\right) \kappa_{1}+4\left(L_{13}+L_{c}\right) f_{1} \kappa_{1}-4\left(L_{35}+L_{c}\right) f_{1} \kappa_{3}} .
\end{gathered}
$$

\section{Chirp control with TDC-based shaping beam line}

A plot of the chirp control for each of the three beam line configurations is shown in Fig. 5. It shows the variation of $R_{56}$ as a function of $\kappa_{1}$ for a 2 TDC $+1 \mathrm{Q}$ and a 3 TDC configuration and $f_{1}$ for a 3 TDC $+2 \mathrm{Q}$ configuration. When the initial longitudinal chirp is $C_{0}$, the chirp after the TDC-based shaping beam line $\left(C_{1}\right)$ can be expressed as

$$
C_{1}=C_{0}+R_{65}
$$

because the bunch length does not change and $R_{66}=1$, where $R$ is the transfer matrix of a shaping system. $R_{65}$ is calculated using linear transfer matrices with a thinlens approximation for quadrupoles. The $2 \mathrm{TDC}+1 \mathrm{Q}$ configuration used $L_{c}=0.3 \mathrm{~m}$ and $L_{12}=L_{23}=1.29 \mathrm{~m}$, and the 3TDC configuration used $L_{c}=0.3 \mathrm{~m}$ and
$L_{12}=L_{23}=1.38 \mathrm{~m}$. The $f_{1}$ and $\kappa$ of other TDCs for these configuration are automatically determined by Eqs. (5), (6), (10), and (11). The 3TDC $+2 \mathrm{Q}$ configuration used $L_{c}=0.3 \mathrm{~m}, L_{12}=L_{45}=1.29 \mathrm{~m}$, and $L_{23}=L_{34}=0.29$. $\kappa_{1}$ and $\kappa_{3}$ for this configuration are fixed to $1 \mathrm{~m}^{-1}$.

The 2TDC + 1Q configuration has the narrowest tuning range, as shown in Fig. 5. This is again due to the absence of a $x-z$ correlation in the incident bunch. On the other hand, the 3TDC configuration provides a wide chirp control range $\left(>30 \mathrm{~m}^{-1}\right)$ compared to the $2 \mathrm{TDC}+1 \mathrm{Q}$ configuration's $<5 \mathrm{~m}^{-1}$.

While the previous two configurations changed $\kappa_{1}$ to control the chirp, the $3 \mathrm{TDC}+2 \mathrm{Q}$ configuration controls the chirp using $f_{1}$. This configuration provided a chirp control range of $\sim 20 \mathrm{~m}^{-1}$ for the focal length change from 0.6 to $4.5 \mathrm{~m}$. This range is comparable to that of the 3TDC configuration, but it does not require changes on $\kappa_{1}$ or 


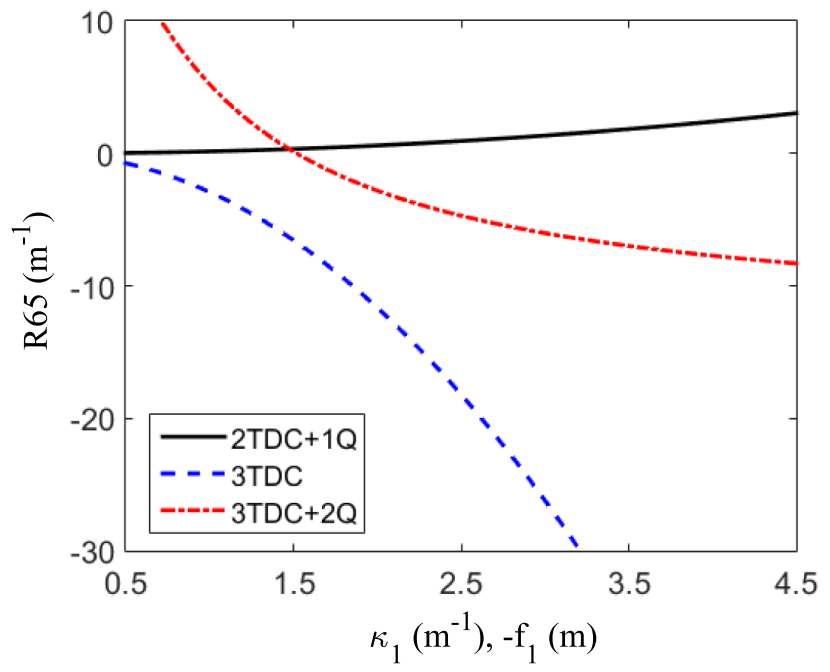

FIG. 5. Change in chirp $\left(R_{65}\right)$ as a function of cavity kick strength $\left(\kappa_{1}\right)$ for a 2 TDC $+1 \mathrm{Q}$ and a 3 TDC configuration and focal length of the first quadrupole $\left(f_{1}\right)$ for a 3 TDC $+2 \mathrm{Q}$ configuration.

element spacing, which both the $2 \mathrm{TDC}+1 \mathrm{Q}$ and the 3TDC configurations require.

This chirp control capability is demonstrated using start-to-end simulation with particle-tracking code GPT. The results of this simulation are discussed in Sec. III.

\section{START-TO-END SIMULATION WITH HIGH-CHARGE BUNCH}

In this section, we perform start-to-end simulations of the TDC-based shaping beam line to characterize its capabilities of arbitrary shaping and chirp control. Later, we discuss two practical issues of the method: bunch length control and charge transmission. We use the GPT code with the Argonne Wakefield Accelerator's (AWA) beam line for the simulation [32]. This start-to-end simulation includes a 3D space-charge effect, and it uses a 3D field map of TDC simulated from CST. Geometric wakefields and CSR are not included in the simulation, because they are negligible in the AWA beam line. Most of the simulations in this section were performed using the $2 \mathrm{TDC}+1 \mathrm{Q}$ configuration, but there is no difference on the shaping performance between the three configurations introduced. This section focuses only on the final results; information on the beam's behavior in the beam line is given in Appendix B.

For the simulation, we chose a uniform distribution with a 16-mm diameter for the laser's transverse parameter and Gaussian distribution with a length of 4-ps FWHM for longitudinal parameters. The gun and linac cavities operated at $1.3 \mathrm{GHz}$, and energy gains were 6.4 and $9.6 \mathrm{MeV}$, respectively. There are a total of six accelerating cavities, so the final energy was around $63 \mathrm{MeV}$. The linac was followed by four quadrupole magnets to control all transverse beam parameters. We located the TDC shaping beam
TABLE I. Beam line parameters for each configuration, which are used for Fig. 5 and all simulations in the paper. Lengths are center-to-center distances.

\begin{tabular}{lcc}
\hline \hline Parameter & Value & Unit \\
\hline$L_{01}$ & 63 & $\mathrm{~cm}$ \\
$L_{m}$ & 109 & $\mathrm{~cm}$ \\
Quad effective length & 10 & $\mathrm{~cm}$ \\
$\kappa_{1}$ & 2 & $\mathrm{~m}^{-1}$ \\
$L_{12}$ & $2 \mathrm{TDC}+1 \mathrm{Q}$ & \\
$L_{23}$ & 118 & $\mathrm{~cm}$ \\
Quad gradient & 118 & $\mathrm{~cm}$ \\
$\kappa_{2}$ & 3.11 & $\mathrm{~T} / \mathrm{m}$ \\
& 2 & $\mathrm{~m}^{-1}$ \\
$L_{12}$ & $3 \mathrm{TDC}$ & \\
$L_{23}$ & & $\mathrm{~cm}$ \\
$\kappa_{2}$ & 118 & $\mathrm{~cm}$ \\
$\kappa_{3}$ & 118 & $\mathrm{~m}^{-1}$ \\
& -4 & $\mathrm{~m}^{-1}$ \\
$L_{12}$ & 2 & $\mathrm{~cm}$ \\
$L_{23}$ & $3 \mathrm{TDC}+2 \mathrm{Q}$ & $\mathrm{cm}$ \\
$L_{34}$ & 118 & $\mathrm{~cm}$ \\
$L_{45}$ & 20 & $\mathrm{~cm}$ \\
Quad1 gradient & 20 & $\mathrm{~T} / \mathrm{m}$ \\
Quad2 gradient & 118 & $\mathrm{~T} / \mathrm{m}$ \\
$\kappa_{2}$ & $1.00,0.75,0.50$ & $\mathrm{~m}^{-1}$ \\
$\kappa_{3}$ & $1.00,0.75,0.50$ & $\mathrm{~m}^{-1}$ \\
\hline \hline
\end{tabular}

line after these quadrupoles. Beam line parameters for simulations in this section are summarized in Table I.

To emphasize the main advantage of the TDC-based shaping over other shaping methods, we chose an initial charge of $60 \mathrm{nC}$. Because of the charge loss through the mask, the bunch charge usually decreases to $20-30 \mathrm{nC}$. Note that most of the shaping simulations and experiments were performed with a total charge of hundreds of picocoulombs [2,4,8,10,11,33]. Only a few EEX-based shaping experiments were performed using 1-2 $\mathrm{nC}$ $[5,34,35]$. A few simulations used only a few nanocoulombs [3,36-38]. The highest charge demonstrated so far was in the simulation for EEX-based shaping using a $10 \mathrm{nC}$ bunch [6]. The shaping for charge level that we show in this paper has never been achieved in either simulation or experiment.

\section{A. Arbitrary shaping for high-charge bunch}

Masking, which is necessary for TDC-based shaping, can shape the longitudinal profile to arbitrary shapes with high accuracy [5]. We applied four different masks to demonstrate the arbitrary shaping capability. Because the WA will be the biggest beneficiary of CSR-free shaping, we chose (a) a doorstep profile, (b) a reversed triangle, (c) a comblike bunch train, and (d) a comblike bunch train with a higher frequency in Fig. 6 as demonstration targets. The actual shape of the mask can be found in Appendix B. This 

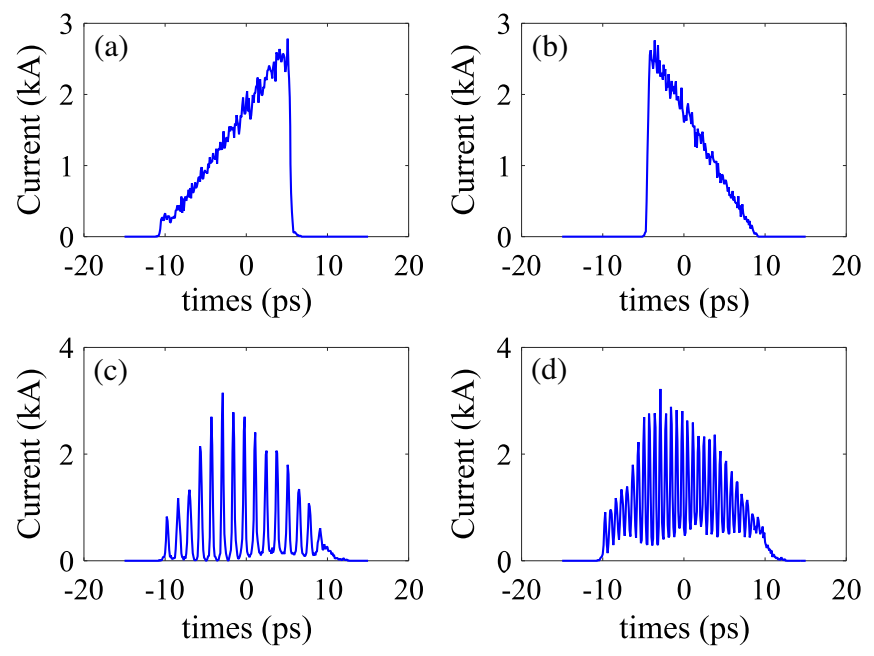

FIG. 6. Start-to-end simulation results for arbitrary shaping using a $60-n C$ bunch. Each panel shows the longitudinal profile after shaping: (a) doorstep, (b) reverse triangle, (c) comblike bunch, and (d) comblike bunch with a higher frequency. The transmitted charges are 22, 18, 12, and $23 \mathrm{nC}$, respectively. Negative time corresponds to the head of the bunch.

Appendix also includes an equation for the mask design. Mask shapes can be achieved by numerically solving this equation.

Figure 6 shows shaped high-charge bunches. The charge started from $60 \mathrm{nC}$, and the mask dropped the charges to $22,18,12$, and $23 \mathrm{nC}$, respectively. Each case successfully generated target profiles, as expected. This result proves the capability to generate arbitrary longitudinal profile. In addition, the TDC-based shaping precisely shaped the bunch to have each target profile. Although it is hard to expect high shaping quality from other methods for such a high charge level, the TDC-based shaping preserved their features, such as the doorstep shape, linear ramp, and bunch-to-bunch separation.

This high-quality shaping is due to the high shaping resolution of the TDC-based shaping. From the simulation, we obtained $\sigma_{x, 0}$ of $3.9 \mathrm{~mm}$. The normalized emittance at this location $\left(\varepsilon_{n, x, 0}\right)$ is $75.9 \mu \mathrm{m}$. According to Eq. (9), we expect that the shaping resolution could be less than $2.37 \mathrm{ps}$ (if $n=3$ ). Again, this number shows the full length of the spread, which may exaggerate its effect on the profile. The actual impact on the profile should be closer to $0.4 \mathrm{ps}$, which is one-sixth of $\Delta z$. In the case of the doorstep and reverse triangle in Figs. 6(a) and 6(b), the tail length (peak to zero) is 1.53 and $0.77 \mathrm{ps}$, respectively. If we define the tail length as the length from peak to $10 \%$ of peak, tail lengths become 0.64 and 0.43 ps. The FWHM of the tail length is 0.35 and $0.21 \mathrm{ps}$; these numbers show reasonable agreement with $\Delta z / 6$, as expected.

Although triangular profiles already demonstrated subpicosecond shaping resolution for high-charge bunches, the bunch train shows this high resolution more clearly, because we can directly observe each bunch's spread. The continuous ramp in the triangular profiles does not respond to the spread if the spread's distribution is symmetric [6]. Figure 6(c) contains a total of 15 microbunches. Because the total charge is $12 \mathrm{nC}$, the charge of each microbunch is a little below $1 \mathrm{nC}$. The bunch length of each microbunch is around 0.5 ps FWHM, which shows good agreement that we obtained from the tail length. As shown in the figure, this charge and bunch length corresponds to a few kiloamperes in current.

In Fig. 6(d), we added more openings on the slit to double the number of microbunches. There are 30 bunches total, and the bunch length is around 0.4 ps FWHM. The charge level is almost same as in Fig. 6(c), but the bunch spacing was halved. Here the microbunch spacing is $0.7 \mathrm{ps}$. This corresponds to $1.4 \mathrm{THz}$. This kiloampere-terahertz bunch train could provide a new opportunity for THz TBA that could potentially generate a gradient of $>1 \mathrm{GV} / \mathrm{m}$. We will discuss applications further in Sec. IV.

\section{B. Chirp control}

The simulation for the chirp control is performed using the same beam line as in Sec. III A. To confirm the tendency in Fig. 5, we tested all three configurations. We kept $\kappa_{1}=2 \mathrm{~m}^{-1}$ for the 2TDC $+1 \mathrm{Q}$ and 3TDC configurations but varied $f_{1}$ for the 3TDC $+2 \mathrm{Q}$ configuration. The quadrupole gradients are 1.00, 0.75, and $0.50 \mathrm{~T} / \mathrm{m}$, which correspond to focal lengths of 2.11 , 2.83 , and $4.26 \mathrm{~m}$, respectively. The final longitudinal phase spaces after the shaping for the doorstep profile are displayed in Fig. 7.

Figure 7(a) is the initial longitudinal phase space, whose chirp is $6.79 \mathrm{~m}^{-1}$. Note that this number is calculated without particles in $t>5 \mathrm{ps}$, because this area will be blocked by the mask. After shaping via the $2 \mathrm{TDC}+1 \mathrm{Q}$ configuration, the chirp changes to $7.24 \mathrm{~m}^{-1}$ (expectation from Fig. 5, $7.39 \mathrm{~m}^{-1}$ ). Because of the overall symmetry, the chirp does not change much in this configuration, as expected. On the other hand, the 3TDC configuration changed the chirp to $-5.35 \mathrm{~m}^{-1}$ (expectation, $-4.85 \mathrm{~m}^{-1}$ ). This configuration can change the chirp dramatically due to the additional TDC.

The 3TDC $+2 \mathrm{Q}$ configuration successfully controlled the chirp with different $f_{1}, \kappa_{1}$ and $\kappa_{3}$ are fixed to $2 \mathrm{~m}^{-1}$, while $f_{2}$ and $\kappa_{2}$ are varied to satisfy the condition in Eqs. (12) and (13). The chirp after the shaping became 2.76, 0.41, and $-2.12 \mathrm{~m}^{-1}$ (expectation, 3.54, 1.29, and $-1.21 \mathrm{~m}^{-1}$ ). We could easily flip the sign of the chirp or zero the chirp using this configuration.

\section{Control of bunch length}

Several of the shaping methods mentioned previously control both the profile and the bunch length simultaneously, but TDC-based shaping does not provide bunch 

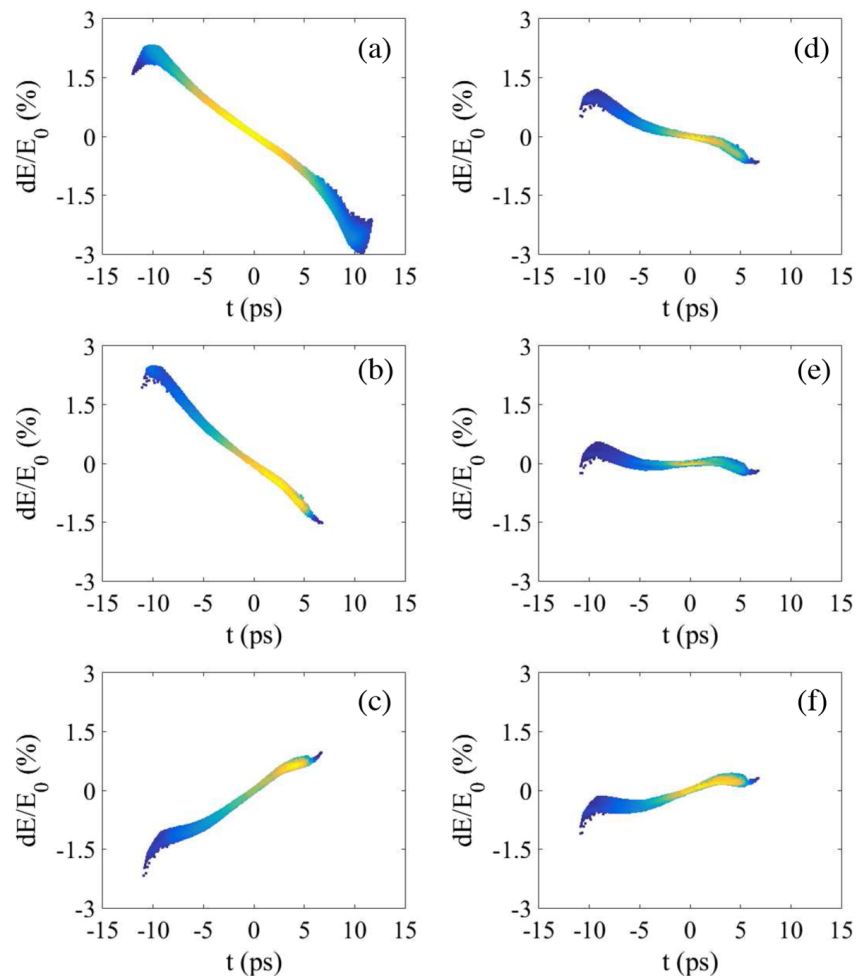

FIG. 7. Start-to-end simulation results to demonstrate chirp control capability. Longitudinal phase space (a) of a 60-nC bunch at the entrance to the shaping beam line and (b)-(f) at the exit to the shaping beam line with a mask for a doorstep profile. (b) and (c) are the results from 2TDC $+1 \mathrm{Q}$ and 3TDC beam lines, respectively. (d)-(f) share the $3 \mathrm{TDC}+2 \mathrm{Q}$ beam line, while they use different first quadrupole strengths: (d) 1.0 , (e) 0.75 , and (f) $0.5 \mathrm{~T} / \mathrm{m}$.

length control. It only introduces a correlation between $z$ and $x$ (or $y$ ), which is not relevant to any of the compression principles (velocity difference and path length difference). There are currently two ways of providing a limited amount of control over the bunch length.

First, one can control the bunch length of the incident bunch. Because the shaping system does not change the bunch length, the final bunch length is proportional to the incident bunch's length if the ratio of the beam size at the mask location to the width of the mask is constant.
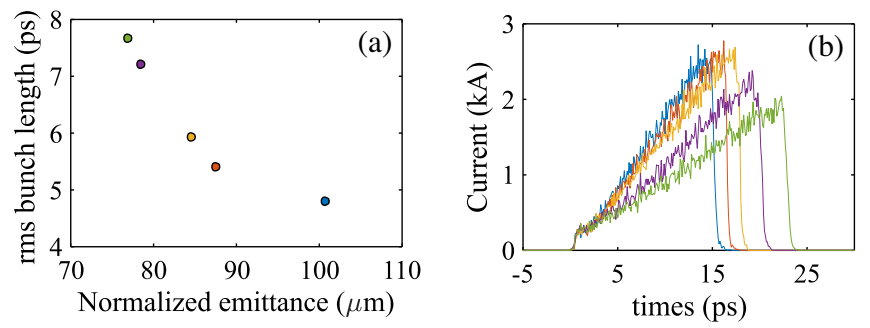

FIG. 8. (a) Simulated trade-off curve between transverse emittance and rms bunch length. (b) Corresponding current profiles.
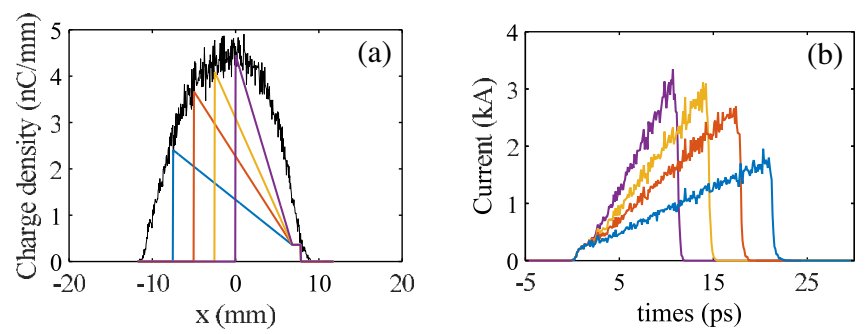

FIG. 9. (a) Horizontal profile at the mask location (black) and shaped horizontal profiles right after the mask (other colors). (b) Final current profiles corresponding to the different mask cut in (a).

Figures $8(\mathrm{a})$ and $8(\mathrm{~b})$ show start-to-end simulation results supporting the claim we made. Here, we varied the pulse length of the laser for photoemission, and we varied the mask width to preserve the ratio. As the initial bunch lengths varies, the final bunch length after the shaping changes correspondingly. The only limitation of this method is the trade-off between bunch length and emittance. As shown in Fig. 8(a), the transverse emittance usually gets worse as the laser pulse gets shorter. In general, a higher emittance means a lower beam quality, and it could drop the shaping resolution to an intolerable level. Similarly, we can imagine applying bunch compression (e.g., chicane) before or after the shaping. However, this introduces a CSR effect to the beam, so it is not preferable.

Second, we can select the cutting range inside the bunch. As shown in Figs. 9(a) and 9(b), the tail location can be selected anywhere inside the bunch while we preserve the profile. This cutting range at the mask will define the final bunch length, as shown in Fig. 9(b). A tail should be located in the $x<0$ region, because a tail located in the $x>0$ region decreases transmission. When the tail is located in $x<0$, the transmission does not change significantly.

Still, the major obstacle to achieve continuous control of the bunch length is a fixed mask. Unless many masks corresponding to each case in Fig. 9(a) are prepared, this method does not provide continuous tuning of the bunch length. One possible candidate to resolve this issue is a multileaf collimator that is commonly used for medical accelerators to shape the electron or x-ray transverse profile. Here, each leaf is controlled by separated motors, so it can form arbitrary transverse shapes. Unfortunately, there are no UHV-compatible multileaf collimators with the precision that shaping beam lines requires. If a technological advance were to enable such multileaf collimators, it would provide not only continuous control of the profile and bunch length, but also real-time feedback for the shape. If it were available, it would have great synergy with this shaping method and emerging machine-learning techniques.

\section{Charge transmission}

The mask used to shape the bunch usually makes a transmission of $50 \%$ or less, depending on the target 

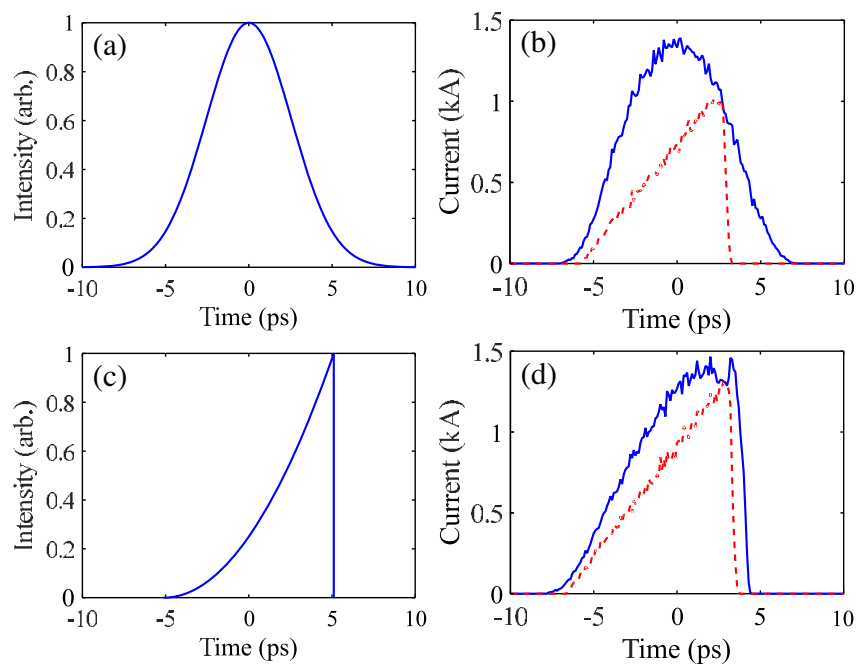

FIG. 10. (a) Gaussian laser profile. (b) Electron beam profiles started from Gaussian laser profile. (c) Shaped laser profile. (d) Electron beam profiles started from the shaped laser profile. Blue and red curves correspond to electron profiles before and after the shaping, respectively. The beam images before and after the mask are given in Appendix B.

profile. Figure 10 shows start-to-end simulations for initially different laser profiles. When the Gaussian-shaped laser generated the bunch, it evolved to a super-Gaussian shape. If we fit a triangular profile to this initial profile, we can get $48.0 \%$ transmission. This is already higher than a typical transmission (30\%). However, to improve this transmission further, we should shape the initial bunch profile (i.e., preshape) to better fit the target profile.

The best candidate for preshaping is the laser shaping technique [1,3]. For a low-charge bunch $(<1 \mathrm{nC})$, laser shaping is the most attractive method due to its simplicity and no-CSR feature. In the case of a low charge, aberration due to $\mathrm{SC}$ should be manageable. However, a high charge $(60 \mathrm{nC}$ in our case) changes the profile significantly, so laser shaping cannot be used alone. The simulation showed that the laser profile in Fig. 10(c) generates a profile deformed by SC like the blue curve in Fig. 10(d). Again, the shaping quality from laser shaping alone is too low to use it for applications such as WAs. However, this deformed profile increased the transmission from $48.0 \%$ to $68.4 \%$. Again, $48 \%$ is the best we can achieve from symmetric profiles, while $68.4 \%$ still has room for improvement.

\section{APPLICATION TO BEAM-DRIVEN WAKEFIELD ACCELERATORS}

In this section, we introduce three WA applications of the TDC-based shaping.

\section{A. Shaped high-charge drive bunch for high transformer ratio}

Transformer ratio $(R)$ is one of the parameters that characterize the performance of WAs. It is defined by the ratio of the maximum decelerating field in the driving bunch to the maximum accelerating field behind the driving bunch. By definition, a high $R$ exploits the driving bunch's energy to accelerate the main bunch. People have tried to increase $R$ by shaping symmetric profiles to asymmetric profiles such as a triangle [39]. So far, three experiments have demonstrated $R \geq 4$ using the shaping [2,34,35].

While these experiments demonstrated a transformer ratio higher than the limitation coming from the symmetry $(R<2)$ [39], these transformer ratios are still much lower than their ideal values. In the case of a doorstep profile, which is the best in practice, the transformer ratio can be expressed as

$$
R(N)=\sqrt{1+(1-\pi / 2+2 \pi N)^{2}},
$$

where $N$ is the ratio of the bunch length to the wavelength. $N$ for the three experiments mentioned earlier is around 2 and corresponds to $R \approx 12$. The measured values from the experiments are less than $50 \%$ of this value. Note that some papers show $R$ higher than $50 \%$ of the ideal, but this depends on which accelerating peak is chosen for the calculation. In practice, the first peak, which has the highest accelerating field, is not appropriate for acceleration due to the smearing from the drive bunch as it loses the energy. Here, we assume that using the second peak is practical. The gap between the ideal transformer ratio and the measured values is the result of a shaping resolution. The simulation demonstrated that $>80 \%$ is achievable [40]. The result in Fig. 6(a) has a similar or higher shaping resolution than the result introduced in Ref. [40], while the charge is approximately 10 times higher.

This high-precision shaping potentially provides two benefits. First, a better profile can generate a higher accelerating gradient. A better profile requires a shorter bunch length than others to achieve the same transformer ratio. When the charge is fixed, it results in a higher gradient. Second, the precise shaping may enable extreme $R$. To achieve $R>20$, for example, the bunch length should be increased. If the shaped profile provides only $50 \%$ of the ideal value, $N$ has to be higher than 6 . If we assume a $100-\mathrm{GHz}$ structure, $N>6$ means the bunch length should be longer than $60 \mathrm{ps}$, which can trigger beam quality and transport issues. On the other hand, a precise shaping that provides $90 \%$ of the ideal value requires $N \approx 3.5$, which may be within a manageable range.

\section{B. Main bunch shaping for high efficiency}

Power efficiency is one of main obstacles to building $\mathrm{TeV}$ linear colliders. Current preliminary designs show that the required power for those $\mathrm{TeV}$ colliders is hundreds of megawatts [41,42], which is not affordable. Several studies are ongoing to improve this efficiency, and the main bunch shaping is one of them. A study on the efficiency in Ref. [43] claims that an ac power requirement for a $\mathrm{TeV}$ 

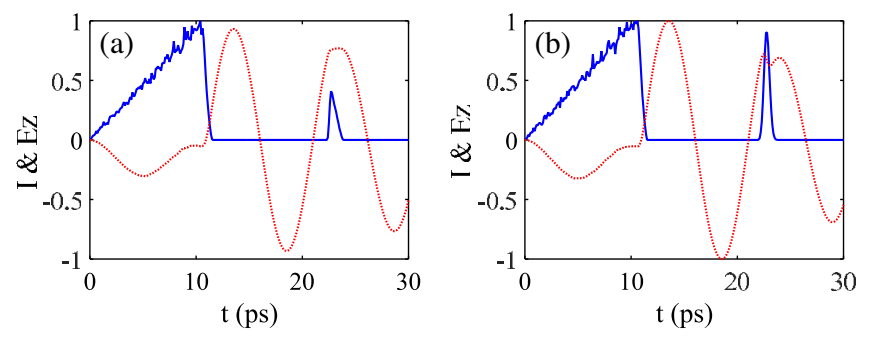

FIG. 11. Current profile (blue) and corresponding wakefield (red). (a) and (b) used the same drive bunch, but the main bunch's profiles are different: (a) reverse triangle and (b) Gaussian.

collider, the Argonne Flexible Linear Collider [42], can be reduced from 297 to $84 \mathrm{MW}$. Here the ac-to-main-beam efficiency is increased from $9.4 \%$ to $33.0 \%$ by assuming an increase of efficiency from klystron, accelerating structure (e.g., dielectric disk structure [43]), and main beam shaping. In the reference, rf-to-main-beam efficiency is doubled by using dielectric disk structure and main beam shaping together.

The main beam shaping improves the rf-to-main efficiency by controlling the beam loading. The energy gain of the main beam is determined by the sum of the accelerating field of the structure and the self-wakefield generated from the main bunch itself. Since the strength of this selfwakefield is proportional to the charge of the main bunch, it is not easy to load high enough charge to efficiently use the power. However, the longitudinal profile of the main bunch can control the shape of this self-wakefield. Figure 11 shows longitudinal profiles of drive and main bunches with corresponding wakefield. Figures 11(a) and 11(b) have different main bunch profiles. Figure 11(b) has a Gaussian main bunch $(0.30 \mathrm{nC})$. Because the particles near the core generates a stronger self-wakefield, the actual accelerating field (red curve) is distorted near the core of the main bunch. This not only limits the maximum charge we can use but also introduces a nonlinearity on the longitudinal phase space and increases the energy spread. On the other hand, the reverse triangle in Fig. 11(a) $(0.52 \mathrm{nC})$ generates a self-wakefield, which is the exact opposite of the accelerating field. In this case, the energy gain along the main bunch becomes flat. We can accelerate more electrons without losing beam quality.

As already demonstrated in Fig. 6(b), the TDC-based shaping can generate this reverse-triangle profile. Combining it with the laser shaping potentially satisfies all the main bunch requirements for future colliders such as profile, charge, and emittance. In addition, this technique can generate both drive and main bunches for collinear wakefield accelerators, as shown in Fig. 11. Because the mask gap determines the temporal separation of bunches, the drive-tomain timing jitter issue in high-frequency wakefield accelerators would benefit from this technique.

\section{High-charge bunch train for THz TBA}

AWA and CLIC are developing the TBA concept for high-gradient acceleration. They are focusing on $1-30 \mathrm{GHz}$ TBA, which provides a much higher accelerating gradient than conventional methods but is reasonably reliable due to its similarity to conventional methods. However, it has a limitation on the achievable accelerating gradient. Because of this limited gradient, it is sometimes considered an intermediate technique between conventional acceleration techniques and plasma-based acceleration techniques.

This limitation on the gradient can be overcome by increasing the operating frequency to THz. Research on $\mathrm{THz}$ structures is receiving increasing attention [44-46] due to the various potential applications of $\mathrm{THz}$ structures. However, most research is performed using laser-based techniques. Here, we introduce a possibility of a beambased technique, THz TBA, to pursue GV/m-class beamdriven acceleration.

One of the main obstacles to realizing THz TBA is the absence of a high-charge bunch train compatible with $\mathrm{THz}$ frequency. Although many techniques have been proposed to generate a $\mathrm{THz}$ bunch train for light-source applications, they cannot generate a high-quality, high-charge bunch train. As we mentioned in Sec. III A, TDC-based shaping is compatible with high-charge bunches, and we already demonstrated the generation of a high-charge bunch train in Fig. 6. In the case of Fig. 6(d), the frequency of the bunch train corresponds to $1.4 \mathrm{THz}$, and the total charge is about $23 \mathrm{nC}$ for 30 bunches. To show that it is possible to reach gigawatt and $\mathrm{GV} / \mathrm{m}$ classes, we made a preliminary design for a 1.4-THz structure and considered two possible bunch train scenarios. Note that the structure is not fully optimized to the beam conditions, and this is only an example. Case 1 used all 30 bunches but cut out charges from each microbunch to make a perfect charge balance between microbunches. In this case, we assume the charge of $0.5 \mathrm{nC}$ per bunch. The other case, case 2, uses only eight bunches, but its charge becomes $1 \mathrm{nC}$. This is achievable, because the bunch in Fig. 6(d) has a higher peak current around the core due to the initial bunch's longitudinal profile. A quartz tube $\left(\epsilon_{r}=3.75, \tan \delta=1 e-3\right)$ is assumed for both the power extraction tube (PET) and accelerating column (ACC).

Table II shows preliminary design parameters for the PET and ACC. Expected power from the PET is $308 \mathrm{MW}$ and $1.2 \mathrm{GW}$, respectively, for case 1 and case 2 . On the accelerator side, the corresponding accelerating gradient reached more than $1 \mathrm{GV} / \mathrm{m}$ for both cases. Case 1 provides $2.0 \mathrm{GV} / \mathrm{m}$, and case 2 provides $4.2 \mathrm{GV} / \mathrm{m}$. These gradients are almost 1 order of magnitude higher than in current TBA schemes. Again, the CSR-free feature of the TDC-based shaping enables the generation of a highcharge bunch train. It may open new opportunities, as described. 
TABLE II. Preliminary $\mathrm{THz}$ TBA design parameters and corresponding power $(P)$ and gradient $(G)$ for each bunch train case.

\begin{tabular}{lcc}
\hline \hline PET parameter & Value & Unit \\
\hline$f_{R F}$ & 1.4 & $\mathrm{THz}$ \\
Inner diameter & 500 & $\mu \mathrm{m}$ \\
Outer diameter & 523 & $\mu \mathrm{m}$ \\
$R / \mathrm{Q}$ & 15.8 & $\mathrm{k} \Omega / \mathrm{m}$ \\
$\mathrm{Q}$ & 1823 & \\
$P_{\text {case 1 }}(30 \times 0.5 \mathrm{nC})$ & 308 & $\mathrm{MW}$ \\
$P_{\text {case 2 }}(8 \times 1.0 \mathrm{nC})$ & 1232 & $\mathrm{MW}$ \\
\hline \hline ACC Parameter & & \\
\hline$f_{R F}$ & Value & $\mathrm{Unit}$ \\
Inner diameter & 1.4 & $\mathrm{THz}$ \\
Outer diameter & 200 & $\mu \mathrm{m}$ \\
$R / \mathrm{Q}$ & 244 & $\mu \mathrm{m}$ \\
Q & 245.8 & $\mathrm{k} \Omega / \mathrm{m}$ \\
$G_{\text {case } 1}(30 \times 0.5 \mathrm{nC})$ & 667 & $\mathrm{GV} / \mathrm{m}$ \\
$G_{\text {case 2 }}(8 \times 1.0 \mathrm{nC})$ & 2.0 & $\mathrm{GV} / \mathrm{m}$ \\
\hline \hline
\end{tabular}

\section{CONCLUSION}

In this paper, we introduced the TDC-based shaping method. This is a new method of shaping a bunch's longitudinal profile that is free from collective effects, which is a major advantage over existing methods. The method is free from CSR, because the shaping process occurs in a straight line. A TDC followed by a drift generates a correlation between $z$ and $x$, a mask is applied for shaping, and then the correlation that remains after shaping can be eliminated by any of the three different configurations we suggested. SC can be ignored if the bunch has high enough energy compared to its charge level.

A limitation of the TDC shaping method is its limited control over the bunch length and low transmission due to the mask. However, it is possible to control the bunch length over a limited range and raise the transmission by combining the method with laser shaping. Despite these limitations, the ability of TDC-based shaping to generate arbitrary profiles with high shaping resolution at high charge (subpicosecond for $20 \mathrm{nC}$ ) is unprecedented. This method will be highly beneficial to beam-driven WAs, as described in the three sample applications. High-charge shaping might allow beam-driven wakefield accelerators to reach the next level.

\section{ACKNOWLEDGMENTS}

This work is supported by the U.S. Department of Energy, Office of High Energy Physics, under Contract No. DE-AC02-06CH11357.

\section{APPENDIX A: ESTIMATION FOR SHAPING RESOLUTION}

In this section, we provide the derivation of Eqs. (7) and (8).

If we consider a two-dimensional Gaussian distribution on an $x-z$ plane, the particle distribution $N(x, z)$ at the mask location without kick from TDC can be written as

$$
N(x, z)=N_{0} \exp \left(-\frac{x^{2}}{2 \sigma_{x}^{2}}-\frac{z^{2}}{2 \sigma_{z}^{2}}\right)
$$

If we ignore particles outside of $\pm n \sigma$, this provides the below equation describing elliptical area in which particles exist:

$$
\frac{x^{2}}{2 \sigma_{x}^{2}}+\frac{z^{2}}{2 \sigma_{z}^{2}} \leq \frac{n^{2}}{2}
$$

If TDC applies a kick to the beam, the particle distribution in Eq. (A1) will change to

$$
N(x, z)=N_{0} \exp \left(-\frac{(x-\kappa L z)^{2}}{2 \sigma_{x}^{2}}-\frac{z^{2}}{2 \sigma_{z}^{2}}\right) .
$$

Similarly, the elliptical area in which particles exist will change to

$$
\frac{(x-\kappa L z)^{2}}{2 \sigma_{x}^{2}}+\frac{z^{2}}{2 \sigma_{z}^{2}} \leq \frac{n^{2}}{2} .
$$

The longitudinal width of this ellipse at a given horizontal position $\left(x_{0}\right)$ will be $\Delta z\left(x_{0}\right)$, as defined in Fig. 2.

From Eq. (A4), $\Delta z$ can be derived as

$$
\Delta z\left(x_{0}\right)=\frac{2 \sigma_{z} \sigma_{x}}{\sigma_{x}^{2}+\left(\kappa L_{m} \sigma_{z}\right)^{2}} \sqrt{\left\{\sigma_{x}^{2}+\left(\kappa L_{m} \sigma_{z}\right)^{2}\right\} n^{2}-x_{0}^{2}} .
$$

Here, $\sigma_{x}^{2}+\left(\kappa L_{m} \sigma_{z}\right)^{2}$ is $\sigma_{m}^{2}$ by definition.

To minimize the resolution, $\sigma_{x}$ must also be minimized. As described in Sec. II, we use a focusing element to minimize $\sigma_{x}$. The minimum value can be written as

$$
\min \left(\sigma_{x}\right)=\frac{\left(L_{m}+L_{01}\right) \varepsilon_{x, 0}}{\sigma_{x, m}},
$$

where $\sigma_{x, 0}$ is the beam size right after the focusing element, as mentioned in Eq. (7). Replacing $\sigma_{x}$ in Eq. (A5) and the definition of $\sigma_{m}$ provides Eq. (7) and Eq. (8), respectively. 


\section{APPENDIX B: BEAM EVOLUTION AND BEAM IMAGE AFTER MASK}

In this Appendix, we show the evolution of the beam along the TDC shaping beam line to more clearly explain the beam's behavior in the beam line. We also provide $x-y$ beam images after the mask for each case given in Sec. III.

Figure 12 shows the projection of the beam to the $x-y$ and $z-x$ planes. This example shows images from the start-to-end simulation result for the doorstep case in Fig. 6. The left and right columns in Fig. 12 show the $x-y$ and $z-x$ projections, respectively. Each row of the figure corresponds to a location where the beam images were taken. The first row corresponds to the entrance of the first TDC. The second and third rows correspond to the entrance and exit of the mask, respectively. The fourth row corresponds to the exit of the shaping beam line.
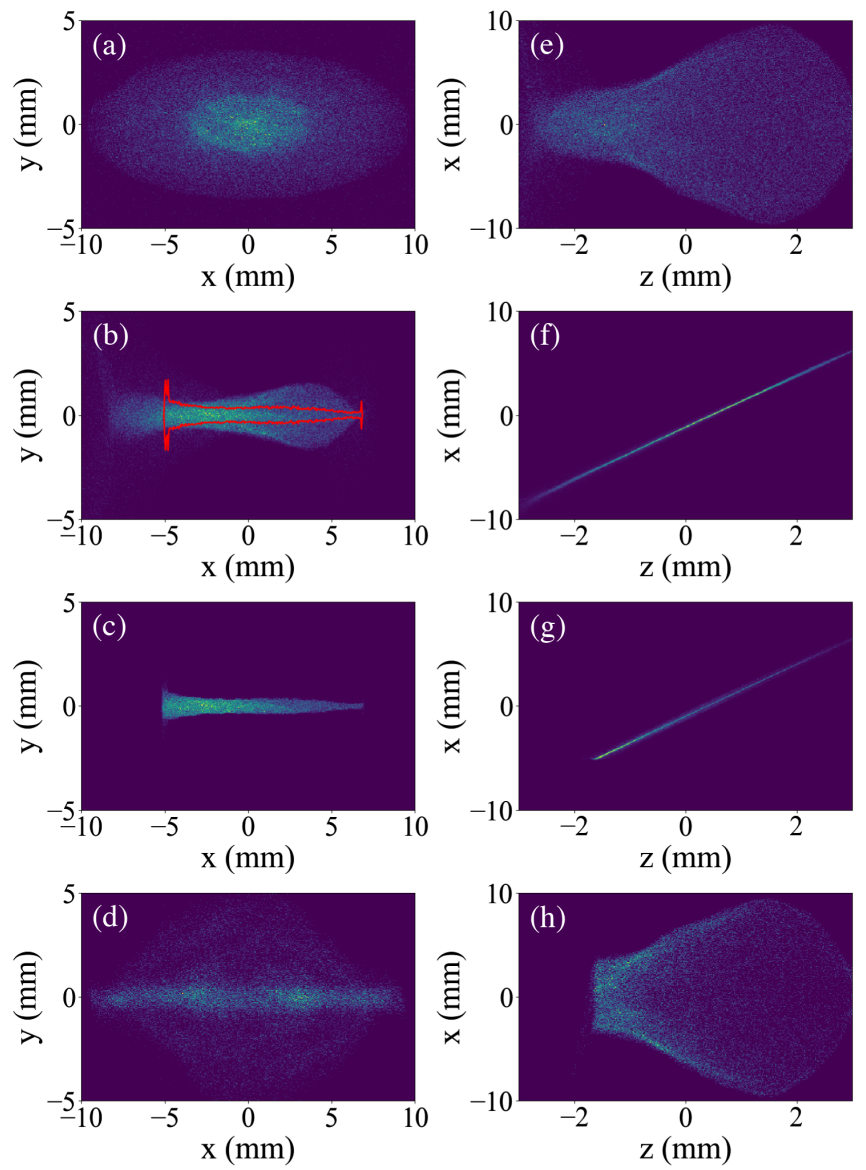

FIG. 12. Beam images on the $x-y$ and $z-x$ plane. Each row corresponds to a location along the shaping system. (a) and (e) are the images at the entrance of the shaping system. (b) and (f) are the images at the entrance of the mask. The red curve in (b) is the shape of the mask used for the simulation. A particle sitting inside the mask area passes through the mask, while other particles are stopped by the mask. (c) and (g) are the images at the exit of the mask. (d) and (h) are the images at the exit of the shaping system. These images are simulation results for the doorstep profile given in Sec. III.
Quadrupole magnets located in front of the shaping beam line focus the beam to minimize the initial transverse contributions [i.e., first two terms of Eq. (2)]. Thus, we can clearly see a linear correlation between $z$ and $x$ at the mask location; see Fig. 12(f). When the beam passes the mask, it is chopped in the $x, y$, and $z$ planes due to the linear correlation. This shaping provides the target profile in the longitudinal direction. The work that remains is to eliminate the correlation the first part of the beam line introduced. Figures 12(d) and 12(h) clearly show that the overall shape returned to its original shape, because the correlation introduced by the first part of the beam line is completely canceled out by the second part of the beam line.

The following two figures show the beam images at the exit of various masks for different cases introduced in Sec. III. Each panel in Fig. 13 corresponds to panels in Fig. 6. In the simulation, particles in positive $x$ are the preceding particles. A multislit used for Fig. 13(d) has the same opening as one for Fig. 13(c), but the number of slits is double of that in Fig. 13(c). Therefore, the openings of the slits are the same in Figs. 13(c) and 13(d), but the number of slits in Fig. 13(d) is double that in Fig. 13(c).

Figure 14 shows the beam images before and after the mask for the laser shaping case described in Sec. III. Figures 14(a) and 14(b) show the beam images before the mask. Although the overall shape is similar, the tail on the negative $x$ is much shorter in Fig. 14(b). In addition, a bright core is shifted to the left in Fig. 14(b) due to the shaped laser profile. Figures 14(c) and 14(d) show the beam images after the mask. Again, they show a similar shape due to similarities in initial distribution. However, for the most part, the beam from the Gaussian laser profile shows a smaller vertical size after the mask compared to the shaped laser case, because the Gaussian laser profile case should
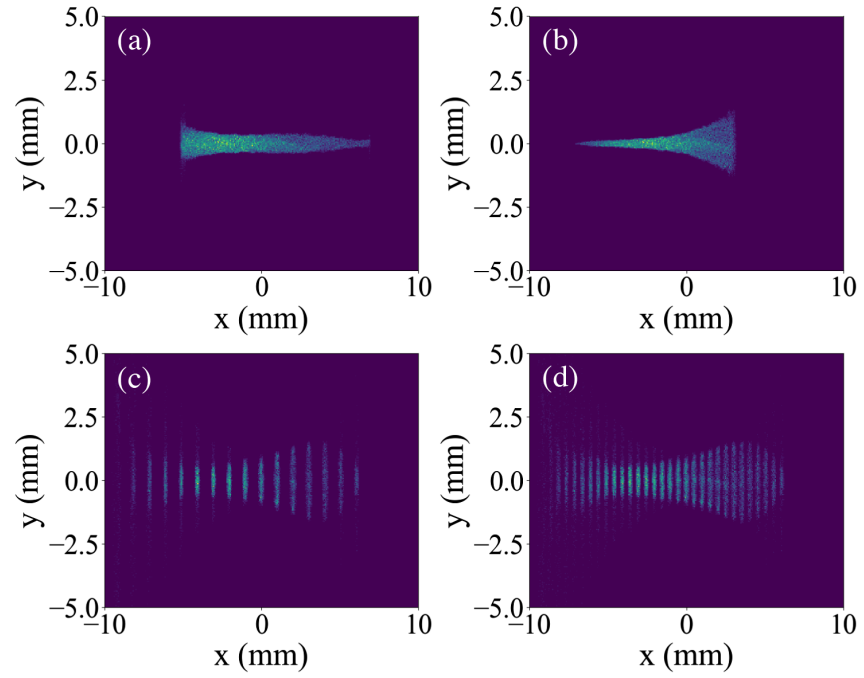

FIG. 13. Beam images after the mask for various profiles in Fig. 6: (a) doorstep, (b) reverse triangle, (c) comblike bunch, and (d) comblike bunch with a higher frequency. 

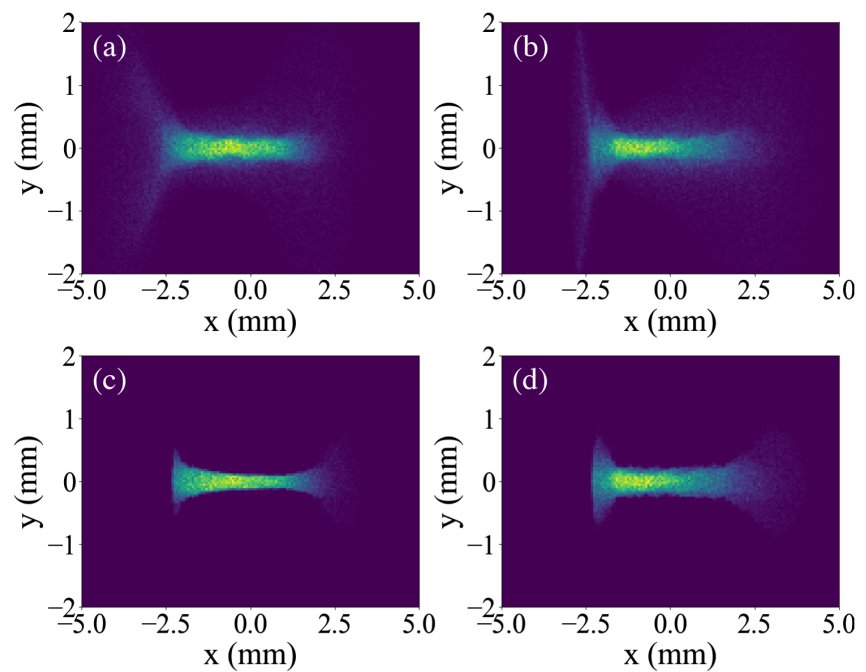

FIG. 14. Beam images (a),(b) before and (c),(d) after the mask. (a) and (c) correspond to the Gaussian laser profile case in Fig. 10. (b) and (d) correspond to the shaped laser profile case.

cut charges near the core of the beam more than the shaped laser case does.

These mask shapes can be easily achieved by solving the equation below:

$$
g(x)=2 \int_{0}^{f(x)} N(x, y) d y,
$$

where $g(x)$ is the target horizontal profile, $f(x)$ is the shape of the mask, and $N(x, y)$ is the beam's transverse distribution at the mask location. Here, we assume symmetry between positive and negative $y$ directions. This is only for the simple design, not the performance.

When solving Eq. (B1), we assumed the use of a multileaf collimator widely used in the medical field. We first decided the width of the leaf and number of leaves. Then, Eq. (B1) tells us the vertical location of each leaf. Although a high-precision, UHV-compatible multileaf collimator has not been developed yet, it will be a very powerful tool when it works with masking-based shaping techniques as described in the main text.

[1] I. Kuzmin, S. Mironov, E. Gacheva, V. Zelenogorsky, A. Potemkin, E. Khazanov, A. Kanareykin, S. Antipov, M. Krasilnikov, G. Loisch, and F. Stephan, Shaping triangular picosecond laser pulses for electron photoinjectors, Laser Phys. Lett. 16, 015001 (2018).

[2] G. Loisch et al., Observation of High Transformer Ratio Plasma Wakefield Acceleration, Phys. Rev. Lett. 121, 064801 (2018).

[3] T. Xu, C. Jing, A. Kanareykin, P. Piot, and J. G. Power, Spatio-temporal shaping of the photocathode laser pulse for low-emittance shaped electron bunches, in Proceedings of IPAC19, Melbourne, Australia (JACoW, Geneva, 2019), pp. 2163-2166.

[4] P. Piot, Y.-E. Sun, J. G. Power, and M. Rihaoui, Generation of relativistic electron bunches with arbitrary current distribution via transverse-to-longitudinal phase space exchange, Phys. Rev. Accel. Beams 14, 022801 (2011).

[5] G. Ha, M. H. Cho, W. Namkung, J. G. Power, D. S. Doran, E. E. Wisniewski, M. Conde, W. Gai, W. Liu, C. Whiteford, Q. Gao, K.-J. Kim, A. Zholents, Y.-E. Sun, C. Jing, and P. Piot, Precision Control of the Electron Longitudinal Bunch Shape using an Emittance-Exchange Beam Line, Phys. Rev. Lett. 118, 104801 (2017).

[6] G. Ha, M. H. Cho, W. Gai, K.-J. Kim, W. Namkung, and J. G. Power, Perturbation-minimized triangular bunch for high-transformer ratio using a double dogleg emittance exchange beam line, Phys. Rev. Accel. Beams 19, 121301 (2016).

[7] G. Ha, J. G. Power, M. Conde, D. S. Doran, and W. Gai, Preparations for installation of the double emittance exchange beamline at the argonne wakefield accelerator facility, in Proceedings of FEL17, Santa Fe, NM (JACoW, Geneva, 2017), pp. 336-339.

[8] R. J. England, J. B. Rosenzweig, and G. Travish, Generation and Measurement of Relativistic Electron Bunches Characterized by a Linearly Ramped Current Profile, Phys. Rev. Lett. 100, 214802 (2008).

[9] P. Piot, C. Behrens, C. Gerth, M. Dohlus, F. Lemery, D. Mihalcea, P. Stoltz, and M. Vogt, Generation and Characterization of Electron Bunches with Ramped Current Profiles in a Dual-Frequency Superconducting Linear Accelerator, Phys. Rev. Lett. 108, 034801 (2012).

[10] G. Andonian, S. Barber, F. H. O'Shea, M. Fedurin, K. Kusche, C. Swinson, and J. B. Rosenzweig, Generation of Ramped Current Profiles in Relativistic Electron Beams using Wakefields in Dielectric Structures, Phys. Rev. Lett. 118, 054802 (2017).

[11] P. Muggli, B. Allen, V. E. Yakimenko, J. Park, M. Babzien, K. P. Kusche, and W. D. Kimura, Simple method for generating adjustable trains of picosecond electron bunches, Phys. Rev. Accel. Beams 13, 052803 (2010).

[12] J. Qiu, G. Ha, C. Jing, S. V. Baryshev, B. W. Reed, J. W. Lau, and $\mathrm{Y}$. Zhu, GHz laser-free time-resolved transmission electron microscopy: A stroboscopic high-dutycycle method, Ultramicroscopy 161, 130 (2016).

[13] Y.-C. Du, W.-H. Huang, and C.-X. Tang, A new method to generate relativistic comb bunches with tunable subpicosecond spacing, Chin. Phys. C 36, 151 (2012).

[14] B. E. Carlsten, K. A. Bishofberger, S. J. Russell, and N. A. Yampolsky, Using an emittance exchanger as a bunch compressor, Phys. Rev. Accel. Beams 14, 084403 (2011).

[15] T. Shintake et al., A compact free-electron laser for generating coherent radiation in the extreme ultraviolet region, Nat. Photonics 2, 555 (2008).

[16] Y. Ding, A. Brachmann, F.-J. Decker, D. Dowell, P. Emma, J. Frisch, S. Gilevich, G. Hays, P. Hering, Z. Huang, R. Iverson, H. Loos, A. Miahnahri, H.-D. Nuhn, D. Ratner, J. Turner, J. Welch, W. White, and J. Wu, Measurements and Simulations of Ultralow Emittance and Ultrashort Electron Beams in the Linac Coherent Light Source, Phys. Rev. Lett. 102, 254801 (2009). 
[17] J. F. Schmerge et al., The LCLS-II injector design, in Proceedings of FEL14, Basel, Switzerland (JACoW, Geneva, 2014), pp. 815-819.

[18] J. H. Lee, J.-H. Han, S.-J. Park, and I. Ko, Slice emittance measurement using rf deflecting cavity at PAL-XFEL ITF, in Proceedings of FEL14 (Basel, Switzerland, 2014), pp. 707-709.

[19] E. Prat, M. Aiba, S. Bettoni, B. Beutner, S. Reiche, and T. Schietinger, Emittance measurements and minimization at the swissfel injector test facility, Phys. Rev. Accel. Beams 17, 104401 (2014).

[20] G. Ha, M. H. Cho, W. Namkung, W. Gai, and P. J. Kim, High-charge-short-bunch operation possibility at Argonne Wakefield accelerator facility, in Proceedings of IPAC15, Richmond, VA (JACoW, Geneva, 2015), pp. 2572-2574.

[21] P. Zhu, Y. Zhu, Y. Hidaka, L. Wu, J. Cao, H. Berger, J. Geck, R. Kraus, S. Pjerov, Y. Shen, R. I. Tobey, J. P. Hill, and X. J. Wang, Femtosecond time-resolved MeV electron diffraction, New J. Phys. 17, 063004 (2015).

[22] S. P. Weathersby et al., Mega-electron-volt ultrafast electron diffraction at SLAC national accelerator laboratory, Rev. Sci. Instrum. 86, 073702 (2015).

[23] F. Brinker, Commissioning of the European XFEL injector, in Proceedings of IPAC16, Busan, Korea (JACoW, Geneva, 2016), pp. 1044-1047.

[24] S. Setiniyaz, H. W. Kim, I.-H. Baek, J. Nam, M. Chae, B.-H. Han, B. Gudkov, K. H. Jang, S. Park, Y. U. Jeong, S. Miginsky, and N. Vinokurov, Beam characterization at the KAERI UED beamline, J. Korean Phys. Soc. 69, 1019 (2016).

[25] C. Gulliford, A. Bartnik, and I. Bazarov, Multiobjective optimizations of a novel cryo-cooled dc gun based ultrafast electron diffraction beam line, Phys. Rev. Accel. Beams 19, 093402 (2016).

[26] N. Neveu, J. Larson, J. G. Power, and L. Spentzouris, Photoinjector optimization using a derivative-free modelbased trust-region algorithm for the Argonne Wakefield Accelerator, J. Phys. Conf. Ser. 874, 012062 (2017).

[27] T. Asaka, H. Ego, H. Hanaki, T. Hara, T. Hasegawa, T. Hasegawa, T. Inagaki, T. Kobayashi, C. Kondo, H. Maesaka, S. Matsubara, S. Matsui, T. Ohshima, Y. Otake, T. Sakurai, S. Suzuki, Y. Tajiri, S. Tanaka, K. Togawa, and H. Tanaka, Low-emittance thermionic-gun-based injector for a compact free-electron laser, Phys. Rev. Accel. Beams 20, 080702 (2017).

[28] C. Joshi, E. Adli, W. An, C. E. Clayton, S. Corde, S. Gessner, M. J. Hogan, M. Litos, W. Lu, K. A. Marsh, W. B. Mori, N. Vafaei-Najafabadi, B. O'shea, X. Xu, G. White, and V. Yakimenko, Plasma wake_eld acceleration experiments at FACET II, Plasma Phys. Controlled Fusion 60, 034001 (2018).

[29] A. Gover, R. Ianconescu, A. Friedman, C. Emma, N. Sudar, P. Musumeci, and C. Pellegrini, Superradiant and stimulated-superradiant emission of bunched electron beams, Rev. Mod. Phys. 91, 035003 (2019).

[30] A. Marinelli, D. Ratner, A. A. Lutman, J. Turner, J. Welch, F.-J. Decker, H. Loos, C. Behrens, S. Gilevich, A. A. Miahnahri, S. Vetter, T. J. Maxwell, Y. Ding, R. Coffee, S. Wakatsuki, and Z. Huang, High-intensity double-pulse x-ray free-electron laser, Nat. Commun. 6, 6369 (2015).
[31] N. Yampolsky, E. I. Simakov, and A. Malyzhenkov, Imposing strong correlated energy spread on relativistic bunches with transverse deflecting cavities, Phys. Rev. Accel. Beams 23, 054403 (2020).

[32] M. Conde, D. S. Doran, W. Gai, W. Liu, J. G. Power, J. Shao, C. Whiteford, E. Wisniewski, S. Antipov, C. Jing, J. Qiu, N. Neveu, Y. Wang, G. Ha, Q. Gao, and L. Zheng, Research program and recent results at the Argonne Wakefield accelerator facility (AWA), in Proceedings of IPAC17, Copenhagen, Denmark (JACoW, Geneva, 2017), pp. 2885-2887.

[33] D. Shchegolkov, E. Simakov, S. Antipov, and M. Fedurin, Dielectric wakefield accelerator experiments at ATF, in Proceedings of IPAC15, Richmond, VA (JACoW, Geneva, 2015), pp. 2681-2684.

[34] Q. Gao, G. Ha, C. Jing, S. P. Antipov, J. G. Power, M. Conde, W. Gai, H. Chen, J. Shi, E. E. Wisniewski, D. S. Doran, W. Liu, C. E. Whiteford, A. Zholents, P. Piot, and S. S. Baturin, Observation of High Transformer Ratio of Shaped Bunch Generated by an Emittance Exchange Beam Line, Phys. Rev. Lett. 120, 114801 (2018).

[35] R. Roussel, G. Andonian, W. Lynn, K. Sanwalka, R. Robles, C. Hansel, A. Deng, G. Lawler, J. B. Rosenzweig, G. Ha, J. Seok, J. G. Power, M. Conde, E. Wisniewski, D. S. Doran, and C. E. Whiteford, Single Shot Characterization of High Transformer Ratio Wakefields in Nonlinear Plasma Acceleration, Phys. Rev. Lett. 124, 044802 (2020).

[36] B. Jiang, C. Jing, P. Schoessow, J. Power, and W. Gai, Formation of a novel shaped bunch to enhance transformer ratio in collinear wakefield accelerators, Phys. Rev. Accel. Beams 15, 011301 (2012).

[37] D. Y. Shchegolkov and E. I. Simakov, Design of an emittance exchanger for production of special shapes of the electron beam current, Phys. Rev. Accel. Beams 17, 041301 (2014).

[38] F. Lemery and P. Piot, Tailored electron bunches with smooth current profiles for enhanced transformer ratios in beam-driven acceleration, Phys. Rev. Accel. Beams 18, 081301 (2015).

[39] K. L. F. Bane and P. B. Wilson, On collinear wakefield acceleration, Report No. SLAC-PUB-3662, 1985.

[40] G. Ha, Q. Gao, E. Wisniewski, J. Power, J. Shao, M. Conde, W. Liu, A. Zholents, C. Jing, and R. Roussel, Observation of high transformer ratio from bunch shaping using emittance exchange, in Proceedings of the 2018 IEEE Advanced Accelerator Concepts Workshop (AAC), Breckenridge, CO (IEEE, New York, 2018), pp. 1-5.

[41] A multi-TeV linear collider based on CLIC technology, Report No. CERN-2012-007, 2012, cLIC conceptual design report.

[42] W. Gai, J. G. Power, and C. Jing, Short-pulse dielectric two-beam acceleration, J. Plasma Phys. 78, 339 (2012).

[43] J. Shao, C. Jing, E. Wisniewski, J. Power, M. Conde, W. Liu, L. Zheng, N. Neveu, D. Doran, C. Whiteford, and W. Gai, Recent progress of short pulse dielectric two-beam acceleration, in Proceedings of IPAC18, Vancouver, Canada (JACoW, Geneva, 2018), pp. 640-643.

[44] E. A. Nanni, W. R. Huang, K.-H. Hong, K. Ravi, A. Fallahi, G. Moriena, R. J. D. Miller, and F. X. Kärtner, 
Terahertz-driven linear electron acceleration, Nat. Commun. 6, 8486 (2015).

[45] D. Zhang, A. Fallahi, M. Hemmer, X. Wu, M. Fakhari, Y. Hua, H. Cankaya, A.-L. Calendron, L. E. Zapata, N.H. Matlis, and F.X. Kärtner, Segmented terahertz electron accelerator and manipulator, Nat. Photonics 12, 336 (2018).

[46] E. Curry, S. Fabbri, J. Maxson, P. Musumeci, and A. Gover, Meter-Scale Terahertz-Driven Acceleration of a Relativistic Beam, Phys. Rev. Lett. 120, 094801 (2018). 